\title{
Determinación del perfil de desempeño de docentes y auditores de ICONTEC y lineamientos para aportar valor a las organizaciones ${ }^{*}$
}

\section{Performance profile determination of ICONTEC teachers and auditors, and guidelines to add value to organizations}

Recibido: 22 de enero de 2014 Revisado: 22 de marzo de 2014 Aceptado: 27 de junio de 2014

Carlos Alberto Díaz Ruiz**

ICONTEC

María Paula López Esguerra***

ICONTEC

\section{RESUMEN}

Alineada con el direccionamiento de ICONTEC, la presente investigación tiene como objetivo principal identificar el perfil y los lineamientos de desempeño de los docentes y auditores con el fin de agregar valor al momento de prestar sus servicios en las organizaciones. Esta investigación está enmarcada en tres enfoques asociados al concepto de agregación de valor: el modelo de liderazgo estratégico y consistente para la mejora continua de las organizaciones (Gámez, Pinzón y Pulido, 2009), la globalización económica y sus beneficios y las recomendaciones de agregación de

\footnotetext{
* Artículo de investigación.

** Carlos Alberto Diaz Ruiz. Ingeniero Industrial, Especialista en Administración de Empresas. Profesional de Certificación de ICONTEC. Correo electrónico: carldiar@hotmail.com.

*** María Paula López Esguerra. Ingeniero de alimentos. Profesional de diseño de servicios de educación de ICONTEC. Correo electrónico: mapelopez70@hotmail.com.
} 
valor en auditorías de sistemas de gestión emanadas de los organismos relacionados con el ICONTEC como la $\mathrm{ISO}^{1}$, el $\mathrm{IAF}^{2}$ y el PTB de Alemania ${ }^{3}$.

Con base en estas premisas se diseña una encuesta que se aplica a los auditores y docentes del instituto a nivel nacional; también se entrevista a los Directores de Educación ${ }^{4}$ y Evaluación de la conformidad ${ }^{5}$ y se realiza una validación de los resultados a través de dos grupos focales con clientes de los servicios de ICONTEC. A partir de estos resultados se determinan los lineamientos de desempeño que definen cuales serían las condiciones que deben gestarse al interior del Instituto para lograr el nivel de desempeño esperado en los profesionales en materia de aporte de valor y se presenta el perfil que debería identificar al tipo de profesional que responde a los elementos identificados desde los enfoques teóricos analizados.

Palabras clave: agregación de valor, auditor, docente, sistemas de gestión.

\section{ABSTRACT}

Aligned with addressing of ICONTEC, this research has as main objective to identify the performance profile and guidelines for teacher and auditors in order to add value when serve in the organizations.

This research is framed on three approaches associated with the concept of value: the model of strategic and consistent leadership for continual improvement of

1 Organización Internacional de Estandarización.

2 Foro Internacional de Acreditación

3 Physikalisch Technische Bundesanstalt.

4 Carlos Pulido Riveros. Director de Educación de ICONTEC.

5 Mónica Vivas Rodríguez. Directora de Evaluación de la Conformidad de ICONTEC. the organizations, economic globalization and its benefits and recommendations of adding value in audits of management systems emanating from ICONTEC related agencies as ISO, IAF and the PTB in Germany.

Based on these premises, is designed a survey that applies to auditors and teachers of the Institute at national level; also interview the Directors of Education and Conformity Assessment and validation of results is conducted through two focus groups with ICONTEC services customers. Performance guidelines, which would define the conditions that must take shape within the Institute to achieve the level of performance expected of practitioners in delivering value are determined, and the profile that should identify the type of professional who responds the elements identified from the theoretical approaches are presented.

Keywords: Value aggregation, auditor, teacher, management systems.

\section{INTRODUCCIÓN}

Tanto en el contexto mundial como en Colombia, la dinámica económica y empresarial impone un reto a los auditores y docentes de los sistemas de gestión para agregar valor en los servicios que prestan a las organizaciones. Independientemente de las razones que motivan a las empresas a certificarse, bien sea por necesidades comerciales o por convicción de los beneficios que aportan los sistemas de gestión, la exigencia a estos profesionales de agregar cada vez mayor valor implica que estén en permanente actualización y mejoramiento de sus competencias técnicas.

En este orden de ideas, el equipo investigador determinó el problema de investigación como el desconocimiento de lineamientos específicos de desempeño 
y perfil de los docentes y auditores de ICONTEC, necesarios para agregar valor a las organizaciones, teniendo como referente teórico las siguientes tres tesis:

- Efectos de la Globalización Económica, según los plantea Amartya Sen (premio Nobel de Economía en1998) (Sen, 2001);

- Liderazgo Estratégico y Consistente para la Mejora Continua Sostenible de las Organizaciones (Gámez et ál., 2009) y,

- Directrices ISO - IAF y PTB para Agregación de Valor en Auditorías.

Derivado de este problema se plantearon los siguientes interrogantes a resolver mediante la investigación:

1) ¿Cuál es la brecha existente entre la metodología actual de desempeño de docentes y auditores de ICONTEC, y las directrices de generación de valor derivadas de los tres enfoques teóricos analizados en la investigación?

2) ¿Qué lineamientos de desempeño se deben establecer para que docentes y auditores de ICONTEC aporten mayor valor a las organizaciones al considerar las directrices emanadas de los tres enfoques teóricos analizados?

3) ¿Cuál debería ser el perfil de docentes y auditores de ICONTEC que se ajuste a los requerimientos de desempeño identificados para la generación de valor?

Los servicios de docencia y de auditoría en sistemas de gestión son los más importantes de ICONTEC; este trabajo aporta al direccionamiento estratégico del Instituto a través de la identificación de nuevos lineamientos para el desempeño y el perfil de auditores y docentes, y busca contribuir a la unificación de criterios al respecto de administrar las competencias que ICONTEC requiere en sus profesionales, dando herramientas para apoyar los planes de selección, desarrollo y mantenimiento de dichas competencias, contribuyendo así a consolidar el posicionamiento que el Instituto ha logrado a lo largo de 50 años de labores al servicio de las empresas del país y de Latinoamérica.

Para el desarrollo del trabajo se plantean las siguientes hipótesis:

1) Los lineamientos de desempeño actuales para auditores y docentes de ICONTEG difieren de lo requerido para agregar valor en las organizaciones con fundamento en las teorías de la globalización y sus beneficios, el liderazgo estratégico y consistente para la mejora continua y sostenible, y la agregación de valor en auditorías.

2) Los perfiles de competencias definidos en ICONTEC para sus auditores y docentes no se ajustan a las características propias de un referente para agregar valor en las organizaciones a partir de los tres enfoques teóricos analizados en la investigación.

Como objetivos se plantearon:

- Identificar la brecha entre la metodología actual de desempeño de docentes y auditores de ICONTEG, y las directrices de generación de valor.

- Definir los lineamientos de desempeño para que docentes y auditores de ICONTEC aporten mayor valor a las organizaciones.

- Diseñar un perfil de docentes y auditores de ICONTEC que se ajuste a los requerimientos de desempeño identificados para la generación de valor. 
Los tres enfoques teóricos utilizados como referente en esta investigación son: en primer lugar la globalización y distribución de sus beneficios, expuesta por Amartya Sen (2001); este enfoque afirma que los beneficios de la globalización se deberían distribuir entre las sociedades y poblaciones más necesitadas y que, en este sentido, a pesar de que la globalización ha traído grandes dividendos y oportunidades para unos cuantos, también ha traído injusticias en la medida en que esa distribución no se hace de una manera equitativa entre los países pobres y ricos que asegure el beneficio de los marginados. Esta tesis sustenta el hecho de que las organizaciones quieran abrirse a nuevos mercados más allá de las fronteras de sus países y ganar un lugar en la oferta internacional, entendiendo que la globalización económica también debe tener efectos sociales positivos que generen desarrollo colectivo y equitativo y deriven en una sociedad abierta (Sen, 2001).

Para ello se requieren prácticas comerciales justas sustentadas en políticas estatales de calidad que busquen compatibilidad técnica y el reconocimiento internacional de estándares de productos y servicios comúnmente implementados y certificados por los organismos de evaluación de la conformidad. En este campo, ICONTEC aporta de manera directa y clara a que una de las restricciones que menciona Sen (2001), como es la restricción comercial, se minimice y sea un pilar para que la distribución de los dividendos de la economía global se efectúe de una manera justa y con equidad para todos los países independientemente de su nivel de desarrollo.

El crecimiento que tiene ICONTEC año tras año en el número de certificaciones emitidas, ${ }^{6}$ se deriva de la ventaja competitiva que las organizaciones ven en la certificación porque les facilita el acceso a otros mercados, ya que los clientes prefieren organizaciones

6 Información suministrada por el Departamento de Mercadeo en agosto de 2012. certificadas que les dan más confianza. Además contar con un sistema de gestión certificado optimiza los procesos, lo que las hace más productivas y finalmente que tengan una mayor rentabilidad por la reducción de costos de producción a partir de menores costos de no calidad como reprocesos, rechazos, fallas y quejas entre otros (ISO Annual Report, 2012).

$\mathrm{Al}$ convertirse ICONTEC en un actor protagónico en la construcción y evaluación de los sistemas de gestión normalizados, así como de los bienes y servicios de las compañías, contribuye de manera definitiva a la competitividad de las organizaciones en un entorno cada vez más globalizado, lo cual a su vez se constituye en un gran reto para sus profesionales al tener que estar actualizados y preparados para responder a las inquietudes y problemáticas que esta dinámica demanda.

En segundo lugar se encuentra la tesis sobre las barreras que impiden la mejora continua de los sistemas de gestión y la propuesta de un modelo de gestión que dé respuesta a esta problemática; el desarrollo de un modelo para la mejora continua sostenible en los sistemas de gestión de las organizaciones, planteado por Gámez et ál. (2009) para los países donde ICONTEC tiene presencia ${ }^{7}$, identifica las barreras que impiden la mejora continua de los sistemas de gestión, una de esas barreras es la falta de liderazgo por parte de la alta dirección de las organizaciones y propone un modelo de gestión que dé respuesta a esta problemática. Para la correcta implementación de la mejora continua sostenible se requiere la aplicación de unos principios que brindan una plataforma cultural; dos de ellos son transversales: el Liderazgo estratégico y consistente y el Enfoque por competencias y participación. También cuenta con tres principios como "pilares técnicos" que permiten sustentar y potenciar el desempeño

7 Actualmente ICONTEC cuenta con oficinas en todos los países de Centro y Suramérica excepto en Argentina, Uruguay, Paraguay y Venezuela. 
integral de la organización los cuales son: Gestión de la información y la comunicación, Enfoque a la innovación y Enfoque a la responsabilidad social (Gámez et ál., 2009).

Esta investigación se centra en el de Liderazgo estratégico y consistente, puesto que tiene que ver directamente con demarcar el rumbo que debería dársele a las organizaciones desde la participación y compromiso con el ser humano en un sistema social en permanente dinámica y aprendizaje colectivo. A continuación se presentan los elementos esenciales de este principio:

De acuerdo con Gámez et ál. (2009), el liderazgo debe ser estratégico, es decir a largo plazo, integral y consistente, donde el líder a través del ejemplo demuestre la coherencia entre lo planificado y lo realizado, siendo este compromiso cada vez mayor con el paso del tiempo y generando una transformación cultural en las organizaciones. Este liderazgo hace referencia a la capacidad de elegir y desplegar una estrategia global en la organización e influenciar y alinear a todas las partes interesadas hacia un mismo fin, motivándolos y comprometiéndolos hacia la acción y haciéndolos responsables por su desempeño y logro de objetivos a largo plazo, donde la mejora continua se convierta en parte de la cultura organizacional.

Un componente importante, según los mencionados autores, del direccionamiento estratégico de una organización es la identificación, difusión e implantación de los valores corporativos que orientan el comportamiento y las decisiones de las personas que hacen parte de esa organización.

Es de resaltar que dentro del direccionamiento estratégico se deben establecer los valores que soporten la mejora continua sostenible, siendo ellos el respeto, el trabajo en equipo, el sentido de pertenencia, la transparencia, la ética empresarial, la autoestima, la perseverancia, el diálogo y el compromiso con las generaciones futuras.

Conforme a Gámez et ál. (2009), el "liderazgo" como un atributo y una competencia propia de quienes dirigen a las organizaciones hacia la mejora continua debe considerarse estratégico y consistente si se quiere maximizar el aporte de los sistemas de gestión en cuanto a prevención y mejora sostenible del desempeño organizacional.

Un segundo elemento a considerar es la relación entre el ejercicio del poder y el estilo de liderazgo que poseen los niveles jerárquicos encargados de tomar las decisiones estratégicas. De allí concluyen que existen barreras en cuanto a saber delegar, saber empoderar a las personas generando sentido de pertenencia y en especial, saber mantener la coherencia entre las directrices impartidas y las decisiones tomadas; no se lidera con base en el ejemplo y no se saben administrar los cambios cuando estos se presentan.

Los docentes y auditores de ICONTEG deberían apoyar el desarrollo de estas competencias en los directivos de las organizaciones clientes, para agregar valor a través del rol que ejercen tanto en las auditorías como en las actividades de formación (Gámez et ál., 2009).

Y por último está la Teoría sobre Agregación de Valor, derivada de las directrices del Grupo de Prácticas de Auditoría de la Organización Internacional de Normalización - ISO y el Foro Internacional de Acreditación - IAF (ISO - IAF, 2009) que junto con las orientaciones dadas por el instituto alemán Physikalisch-Technische Bundesanstalt - PTB, se constituyen en lineamientos teóricos para el desempeño de auditores y docentes de ICONTEC. El conocimiento previo sobre agregación de valor se fundamenta en dos pilares específicos del desempeño de los profesionales de ICONTEC. Por un lado, en el año 2007, el instituto PTB de Alemania desarrolló un 
seminario de recalificación de auditores de ICONTEC resaltando la necesidad de que los auditores fortalezcan sus competencias para agregar valor a las organizaciones y hacer que se esmeren por la mejora continua y sostenible de sus sistemas de gestión. Hacer que el profesional de ICONTEC cuente con las competencias para generar procesos de cambio en las organizaciones exige especialmente su focalización en la parte humana de los sistemas de gestión y la cultura organizacional, que es la base de las iniciativas de mejora (PTB, 2007).

La PTB, en sus memorias, se basa en gran parte en lo expuesto en el capitulo 7 de la norma ISO 19011, la cual trata sobre la competencia y evaluación de los auditores (ICONTEC, 2012).

Al final estas memorias de la PTB concluyen en dos sentidos, por un lado las posibilidades de mejoramiento y por el otro las experiencias exitosas de auditoría. Con respecto a las situaciones que deberín evitarse en el proceso de auditoría se enuncian las siguientes: "gotas de sudor" del auditado, miradas en busca de ayuda, preguntas no entendidas, discusiones interminables, demasiado amistoso y demasiado simpático o eufórico.

En cuanto a experiencias exitosas se dan cuando se obtienen resultados como alto flujo de información desde el auditado, relaciones positivas, atmósfera distensionada, invitación para otra auditoría, comprobación de cambios positivos, se despierta el interés y proceso de mejoramiento continuo (ICONTEC, 2012).

Por otra parte, en los documentos emitidos por el Grupo de Prácticas de Auditoría ISO 9001, liderado por la Organización Internacional de Normalización -ISO y por el Foro Internacional de Acreditación -IAF, en su edición del 4 de junio de 2009, se exponen directrices sobre cómo agregar valor durante el proceso de auditoría. Se aborda el análisis de la agregación de valor desde la perspectiva de dos preguntas fundamentales: ¿qué significa realmente agregar valor?, ¿es posible agregar valor sin comprometer la integridad de la auditoría ni hacer consultoría? en principio, todas las auditorías deben agregar valor, pero este no siempre es el caso.

Según el Grupo de Prácticas de Auditoría (2009), el valor se le confiere a algo que es útil y agregar valor significa hacer que algo sea más útil. Entonces auditores y docentes son sujetos activos en esta propuesta de agregar valor por cuanto su ejercicio, tanto de auditoría como de docencia, debe ser cada vez más útil para el mantenimiento y mejora de los sistemas de gestión en beneficio de sus diferentes partes interesadas.

En este sentido, los auditores como los docentes se deben preparar continuamente observando que su ejercicio se enfoque según el nivel de madurez de los sistemas de gestión de las organizaciones atendidas; por un lado, están aquellas en las cuales sus sistemas de gestión agregan valor porque contribuyen a la forma de hacer negocios, siendo útiles para el logro de los objetivos estratégicos de negocios; por otro, están aquellas organizaciones en las cuales el sistema de gestión se convierte solamente en un conjunto burocrático de procedimientos, documentos y registros que no agregan valor porque no reflejan la realidad de la compañía y lo único que agregan es sobrecostos inútiles a la gestión (Grupo de Prácticas de Auditoría, 2009).

Derivado del análisis de los tres anteriores enfoques, se definieron las premisas a trabajar como variables e indicadores en las encuestas y entrevistas.

\section{METODOLOGÍA}

De acuerdo con Mario Tamayo y Tamayo (2003), según su propósito o finalidad esta investigación es aplicada, ya que se pretende que sus resultados se 
pongan en práctica en ICONTEC O según su alcance, la investigación es descriptiva puesto que caracteriza el objeto de estudio, pretendiendo explicar y describir las características de los auditores y docentes de ICONTEC y sus prácticas profesionales en la prestación de los servicios y desde el punto de vista del método empleado, para Bonilla-Castro y Rodríguez (2005), es una investigación mixta ya que se apoya en datos medibles y cuantificables que se recopilan mediante encuestas a una muestra de profesionales auditores y docentes de ICONTEC, y entrevistas a dos directivos del Instituto con el fin de indagar su opinión acerca de su percepción y conocimientos sobre las características identificadas en las premisas de los tres enfoques teóricos de referencia.

El universo estuvo compuesto por un grupo de 83 profesionales a nivel Colombia, distribuidos entre 62 auditores, profesionales del área de Certificación vinculados de planta al Instituto y 21 docentes, profesionales del área de Educación, igualmente con vinculación directa (ICONTEC- Dirección de Gestión Humana, 2013); es importante resaltar que muchos de estos profesionales ejercen ambos roles, tanto de auditor como de docente lo cual se considera en el diseño de las encuestas.

Antes de aplicar las encuestas y entrevistas se validaron a través de una prueba piloto con el soporte de dos docentes y dos auditores de ICONTEG realizando los ajustes pertinentes para hacer las preguntas más entendibles y comprensibles. El cálculo del tamaño de la muestra se realizo mediante muestreo probabilístico; considerando que el tamaño de la población es de 83 profesionales entre docentes y auditores, con un nivel de confianza del $89 \%$ y un margen de error del $6 \%$, se determina la aplicación de 46 encuestas.

\section{RESULTADOS Y DISCUSIÓN}

Como características de la población estudiada se identifica que 22 de los encuestados son exclusivamente auditores, 7 solo docentes, y los 17 restantes ejercen los dos roles. La Tabla 1 muestra esta proporción.

Tabla 1. Rol que desempeñan los profesionales encuestados en ICONTEC (\%)

\begin{tabular}{|l|c|c|}
\hline \multicolumn{1}{|c|}{ Rol en ICONTEC } & $\begin{array}{c}\text { Cantidad } \\
\text { profesionales }\end{array}$ & $\%$ \\
\hline Solo auditor & 22 & 47,83 \\
\hline $\begin{array}{l}\text { Auditor - docente y docente - } \\
\text { auditor. Doble rol }\end{array}$ & 17 & 36,96 \\
\hline Solo docente & 7 & 15,22 \\
\hline Total & 46 & 100,00 \\
\hline
\end{tabular}

Fuente: elaboración propia.

La distribución de docentes por tiempo de vinculación muestra que la mayoría de estos supera los tres años en el Instituto. Ver Tabla 2.

Tabla 2. Tiempo de vinculación de docentes (\%)

\begin{tabular}{|l|c|c|}
\hline Tiempo de vinculación docentes & $\begin{array}{c}\text { Cantidad } \\
\text { profesionales }\end{array}$ & $\%$ \\
\hline Superior a tres años & 6 & 85,71 \\
\hline Entre uno y tres años & 1 & 12,24 \\
\hline Inferior a un año & 0 & 0,00 \\
\hline Total & 7 & 100,00 \\
\hline
\end{tabular}

Fuente: elaboración propia.

Similar situación se observa en cuanto al tiempo de vinculación con ICONTEC para los auditores, en donde también la mayoría de estos supera los tres años en el Instituto. Ver Tabla 3. 
Tabla 3. Tiempo de vinculación de auditores (\%)

\begin{tabular}{|l|c|c|}
\hline \multicolumn{1}{|c|}{ Tiempo de vinculación } & $\begin{array}{c}\text { Cantidad de } \\
\text { profesionales }\end{array}$ & $\%$ \\
\hline Superior a tres años & 15 & 68,18 \\
\hline Entre uno y tres años & 6 & 27,27 \\
\hline Inferior a un año & 1 & 4,54 \\
\hline Total & 22 & 100,00 \\
\hline
\end{tabular}

Fuente: elaboración propia.

Quienes ejercen el doble rol: auditor -docente y docente- auditor son en su gran mayoría aquellos profesionales que llevan más de tres años de vinculación con el Instituto, pudiendo observarse que esta condición en general la alcanzan después de acumular conocimientos y experiencias suficientes en el rol inicial. Ver Tabla 4.

Tabla 4. Tiempo de vinculación de profesionales auditores-docentes (doble rol) (\%)

\begin{tabular}{|l|c|c|}
\hline \multicolumn{1}{|c|}{ Tiempo de vinculación } & $\begin{array}{c}\text { Cantidad de } \\
\text { profesionales }\end{array}$ & $\%$ \\
\hline Superior a tres años & 16 & 94,12 \\
\hline Entre uno y tres años & 1 & 5,88 \\
\hline Inferior a un año & 0 & 0,00 \\
\hline Total & 17 & 100,00 \\
\hline
\end{tabular}

Fuente: elaboración propia.

Del análisis de los resultados obtenidos de la aplicación de las encuestas a los profesionales de las áreas de Educación y Evaluación de la conformidad, de las entrevistas realizadas a los Directores de estas Unidades estratégicas de negocio y de la validación de los resultados con dos empresas clientes del Instituto, fue posible confirmar las dos hipótesis planteadas; en la primera se asume que los lineamientos de desempeño actuales de auditores y docentes de ICONTEC difieren de lo requerido para agregar valor y en la segunda, se plantea que los perfiles actuales definidos para auditores y docentes de ICONTEC no cubren los referentes teóricos de generación de valor identificados a partir del análisis documental de los tres enfoque teóricos.

Asimismo con los resultados obtenidos, se determinaron los lineamientos específicos para el desempeño y el perfil del docente y auditor con el objetivo de agregar valor en los servicios. Los principales lineamientos determinados se mencionan a continuación:

1. Proporcionar mayores conocimientos e información que le permita a los profesionales sentirse completamente seguros y tranquilos de contar con la competencia suficiente con respecto a los factores socio-económicos, culturales, regulatorios y normativos que afectan a las organizaciones en las cuales prestan sus servicios.

2. Tanto en los planes de carrera que lidera dentro de ICONTEC el área de Gestión Humana, como en los grupos primarios y reuniones técnicas de las Unidades Estratégicas de Negocio, es importante incluir una serie de cursos teórico-prácticos, dirigidos no solo a profesionales sino a directivos, que tengan como propósito formar lideres, con base en los elementos del modelo de Liderazgo estratégico y consistente para la mejora continua y sostenible de las organizaciones.

3. En especial para los profesionales nuevos se debería, durante su inducción y entrenamiento en el primer año, fortalecer las competencias de liderazgo y desarrollar sus habilidades para abordar con propiedad y suficiencia a las personas de la alta dirección de las organizaciones. $\mathrm{Al}$ abordar temas como direccionamiento estratégico y relacionamiento con grupos de interés, el profesional debe ser muy convincente, y a la vez diplomático, al transmitir el mensaje mostrando los beneficios sin demeritar lo propio de la organización visitada. 
4. En torno al desarrollo del profesional, hay que migrar hacia un perfil de prestador de servicios integral, un profesional de talla mundial que no se quede todo el tiempo auditando o dictando solamente, sino cuya carrera en el Instituto comience por Normalización, continúe en Evaluación de la Conformidad y se consolide en Educación, por cuanto esto potencializaría mejor el desarrollo de sus competencias. Se puede comenzar por hacer que los profesionales actuales, que en su mayoría son auditores, se califiquen como docentes y viceversa, que los docentes se califiquen también como auditores. Esto puede hacerse extensivo a los profesionales de Normalización ${ }^{8}$ que pudieran ir dedicando más tiempo a auditar y ejercer la docencia.

5. Asimismo, debe trabajarse en las competencias comportamentales del profesional especialmente la parte actitudinal que debe siempre estar encaminada a una disposición de servir con humildad, con dinamismo y empatía, eliminando toda posibilidad de prepotencia o soberbia con respecto al receptor del servicio. Preparar al profesional para aceptar la realidad que está observando sin caer en críticas destructivas, imprudencia o desviaciones de los objetivos de su curso o de su auditoría.

6. Por otra parte, la programación de los servicios resulta crucial para permitirle al profesional prepararse muy bien y esto incluye asegurarle los tiempos de planificación, en el caso de las auditorías, y el suministro de toda la información previa para este propósito. Para el caso de los servicios de educación, aunque estos no requieren elaborar un plan como tal, si es recomendable que el profesional

8 La Unidad Estratégica de Normalización es el área encargada de desarrollar el proceso de Normalización que junto con el de Evaluación de la Conformidad y Educación hace parte de los procesos relacionados con el servicio de ICONTEC. siempre tenga acceso a la información preliminar de la organización que va a visitar.

7. Para potencializar la motivación en los profesionales que aportan valor es recomendable desarrollar dos actividades fundamentales: la primera consiste en identificar características y capacidades de las personas en relación con el enfoque de agregar valor, es decir, implica preguntarse si la persona tiene las capacidades adecuadas para las exigencias dadas desde los retos del entorno socio-económico que rodea a las organizaciones, desde el enfoque de liderazgo estratégico y consistente necesario para la mejora continua, y por último, desde las directrices de aporte de valor dadas por los organismos aliados a ICONTEC, lo cual quedaría plasmado en el perfil del cargo o descripción del puesto de trabajo. La segunda actividad es valorar y premiar la gestión de estos profesionales a través de la evaluación del desempeño cuyos resultados, a su vez, se convierten en insumo para los planes de formación correspondientes.

\section{CONCLUSIONES}

Se confirman las hipótesis planteadas ya que se pudo establecer que existe una brecha entre el actual desempeño de los docentes y auditores de ICONTEC y los planteamientos derivados de los tres enfoques teóricos analizados, en relación con la agregación de valor durante la prestación de los servicios; además los actuales perfiles de estos profesionales, analizados desde el punto de vista práctico, tampoco se orientan a la agregación de valor.

Al Identificar las brechas metodológicas es posible generar propuestas de lineamientos y perfil; se pudo establecer que el aporte de valor por parte de los profesionales depende en gran medida de cómo el Instituto los rodea de las condiciones necesarias y suficientes para hacer 
que el ejercicio de dictar un curso o realizar una auditoría sea enriquecedor para las partes que intervienen.

$\mathrm{Al}$ someter los tres enfoques teóricos analizados a lo largo de este estudio al escrutinio de diferentes grupos de interés, incluidos los propios profesionales, se han podido identificar lineamientos clave tanto del entorno en el que se desempeña el profesional como de su propia condición personal y laboral, los cuales ameritan ser considerados por parte de ICONTEC para lograr servicios con mayor aporte de valor a las organizaciones. Uno de los lineamientos más importantes, para asegurar el aporte de valor agregado durante la prestación de sus servicios, es que los profesionales desarrollen un plan de carrera que incluya su paso por los procesos de las áreas de Normalización, Certificación y Educación, lo cual enriquecería su visión de la realidad de las organizaciones.

Los resultados de la investigación permiten argumentar la tesis de que los docentes y auditores aportan mayor valor a las organizaciones si cuentan con los lineamientos de desempeño y el perfil de competencias que se derivan de los fundamentos postulados en los tres enfoques teóricos que se toman como referencia de la investigación.

Como aspectos adicionales para complementar la propuesta de perfil y lineamientos, es importante que desde el área de Gestión Humana del Instituto, se exploren teorías adicionales sobre sicología del comportamiento, donde se identifique cómo los estados de ánimo del personal pudieran estar influyendo en el desempeño con aporte de valor, para identificar lineamientos adicionales que contribuyan a enriquecer la propuesta.

\section{REFERENCIAS}

Bonilla-Castro, E. y Rodríguez, P. (2005). Más allá del dilema de los métodos. La investigación en ciencias sociales. Bogotá, D.C.: Universidad de los Andes. Grupo Editorial Norma.

Gámez, S., Pinzón, J. y Pulido, C. (2009). Desarrollo de un modelo para la mejora continua sostenible en los sistemas de gestión de las organizaciones. Tesis de Magister en Calidad y Gestión Integral. Bogotá: Universidad Santo Tomas de Aquino Convenio ICONTEC.

Grupo de Prácticas de Auditoría. (2009). Directrices sobre: Cómo agregar valor durante el proceso de auditoría. Ginebra: ISO e IAF.

Hamburger Fernández, Á. (2008). Los valores corporativos en la empresa, el concepto del valor. Bogotá D.C.: Paulinas.

Instituto Colombiano de Normas Técnicas y Certificación ICONTEG. (2013). Dirección de Gestión Humana. Listado consolidado de profesionales de certificación sistema. Bogotá.

Instituto Colombiano de Normas Técnicas y Certificación ICONTEC. (2012). Directrices para la auditoría de sistemas de gestión NTG - ISO 19011:2012. Bogotá: el Instituto.

Organización Internacional de Normalización. (2012). ISO Annual Report 2011. Ginebra: ISO Central Secretariat.

Physikalisch-Technische Bundesanstalt PTB. (2007). Documentos de trabajo del seminario de recalificación de auditores efectuado en ICONTEC. Bogotá.

Sen, A. (2001). Juicios sobre la globalización, Fractal $\mathrm{N}^{\circ}$ 22, julio-septiembre, volumen VI. 2.

Tamayo y Tamayo, M. (2003). El proceso de la investigación científica. México DF.: Limusa Noriega Editores, cuarta edición. 